\title{
The role of the entrepreneur in health care delivery: the Geisinger Health System - xG Health Solutions example
}

This article was published in the following Dove Press journal:

Innovation and Entrepreneurship in Health

I June 2015

Number of times this article has been viewed

\author{
Earl P Steinberg' \\ Glenn D Steele Jr2 \\ 'xG Health Solutions, Columbia, MD, \\ ${ }^{2}$ Geisinger Health System, Danville, \\ PA, USA
}

\begin{abstract}
The Geisinger Health System (GHS) is a well-established, not-for-profit enterprise that delivers health care and provides health insurance. Having started reengineering the way it delivers care nearly two decades ago, GHS is one of a handful of entities that is well prepared for a shift from fee-for-service to value-based payment. In 2009, GHS' Board of Directors added to GHS' mission the "scaling and generalizing" of the approaches GHS has employed to distinguish itself as a model for how health care should be delivered. This expanded mission was based in part on a desire to become a leader in helping other health care providers improve their clinical and financial performance. Given the increasingly challenging health care payment environment, a decision was made to try, in the course of pursuing this goal, to create a financial return for GHS on the substantial investments GHS had made in innovations over the past two decades. Because of a belief that generalizing, scaling, and commercializing products and services are fundamentally different from delivering health care, and a recognition that GHS employees lacked the time and resources to scale and generalize, GHS created a free-standing, for-profit entity - xG Health Solutions - funded in large part by a venture capital firm, to commercialize GHS intellectual property. This entrepreneurial venture has proven that the tools and methods used at GHS can be adapted and used to help other health care delivery systems reduce the cost and improve the quality of the care they deliver. Although it is too soon to know how successful $\mathrm{xG}$ Health Solutions will be, it has grown substantially during its first 2 years, and appears to have confirmed that GHS' judgment that a separate, but related, entrepreneurial entity was a good way to "scale and generalize" GHS' health care performance improvement intellectual property.
\end{abstract}

Keywords: entrepreneurial, intellectual property, health care, value-based payment, quality of care

\section{Introduction}

Well-established enterprises and entrepreneurial ventures play very important, but different, roles. The former tend to focus on what they have been doing and on maintaining their existing position in a market. ${ }^{1}$ Although well-established enterprises are expected to grow, they often are not successful at driving growth through innovation, in part because they assign responsibility for such growth to managers who are skilled at sustaining current activities and/or operating units whose top priority is to execute successfully on current activities. ${ }^{2}$ Entrepreneurial ventures, in contrast, focus their energy on developing and selling new products and services, and attract individuals who are willing to expend extraordinary effort in order to change the way things are done currently and to take financial risk in return for uncertain, but potentially higher, future financial return.
Correspondence: Earl P Steinberg xG Health Solutions, 6750 Alexander Bell Drive, Suite 200, Columbia, MD 2I046, USA

Tel +l 4432034410

Fax + I 443276672 I

Email esteinberg@xghealth.com 
The Geisinger Health System (GHS) is a well-established, not-for-profit enterprise that delivers health care and provides health insurance, primarily to people living in central and northeastern Pennsylvania. GHS has a strong national reputation not only for delivering high-quality care but also for innovating better ways to deliver health care. Until recently, GHS' focus has been exclusively on delivering health care in the Pennsylvania market. Five years ago, however, GHS' Board of Directors added to Geisinger's mission the "scaling and generalizing" of the approaches Geisinger has employed to distinguish itself as a model for how health care should be delivered. GHS has pursued this "scaling and generalizing" objective in three ways: 1) by acquiring hospitals, provider groups, or health systems that were operating in markets in which Geisinger already operated (eg, Scranton and Shamokin, Pennsylvania) or in different markets (such as New Jersey), 2) by forming joint ventures or other types of partnerships with entities in other markets (such as West Virginia, Maine, and New Jersey), and 3) by launching a new, for-profit company that would operate outside of GHS whose purpose was to export to other health care delivery systems things Geisinger had developed and learned in order to improve the performance of those systems. This paper describes that new enterprise, Geisinger's rationale for establishing it, the approach Geisinger's leadership took to develop it, and its first 2 years of experience.

\section{Rationale for scaling and generalizing}

There were several rationales for incorporating "scaling and generalizing" into Geisinger's mission. One was that it was consistent with the desire of GHS' Board for Geisinger to be a leader - nationally, as well as locally and regionally. Another was a sense of responsibility - since GHS had demonstrated that its innovative approaches to health care delivery had resulted in improved quality and reduced total cost of care, ${ }^{3-6}$ GHS' CEO felt that GHS had a responsibility to help ameliorate deficiencies in other health care delivery systems by exporting to them innovations that Geisinger had employed to address similar challenges at Geisinger.

Third, the health care system in the USA was entering a period of unprecedented turmoil - and opportunity - that Geisinger was well positioned to address. The ultimate payers for health care (federal and state governments, employers, patients) as well as health care providers (eg, hospitals, physicians, and post-acute care providers, such as skilled nursing facilities and home health care companies) increasingly recognized that trends in health care costs were unsustainable and that neither payers nor patients were getting good value for the dollars currently being expended on health care. More importantly, as a result of the enormous pressures being created by health care costs, many payers and providers had come to believe that we had reached a financial tipping point which was going to result in substantial reductions in fee-for-service payment rates as well as movement away from fee-for-service to more bundled and performance-based payment methods. Anticipation of changes in how health care would be paid for, in turn, was pushing an increasing number of health care providers to begin to change how they are organized and how they deliver health care. Having started down a path toward reengineering the way it delivers care nearly two decades earlier, Geisinger was one of a handful of entities that were prepared for the new health care economic environment. Geisinger recognized that most providers lacked the expertise required to transform themselves from a "volume" to a "value" orientation. This situation provided an attractive business opportunity for which Geisinger was well positioned - namely to assist health care providers who want to prepare themselves for and operate successfully in a value- rather than volume-oriented payment environment. By doing so, Geisinger was in a position to create a financial return on the substantial investments Geisinger had made in innovations over the past two decades, a return that could help supplement what Geisinger's CEO thought would be slower growth in revenue that all health care providers were likely to experience over the next decade.

A fourth rationale was to address skepticism that was prevalent in some circles about whether innovations that worked at Geisinger could be replicated elsewhere or whether they were dependent on various signature aspects of Geisinger - such as its culture or the sophisticated customizations GHS has made to its electronic health record (EHR) - that would be very difficult to reproduce.

A final rationale was a hypothesis on the part of Geisinger's CEO that, given recent advances in technology and data analytic science, a new entrepreneurial venture could build a health information technology/data analytic infrastructure that would be more efficient and have more capabilities than the one Geisinger had developed incrementally over the past two decades. If that were the case, Geisinger's CEO thought that the new entrepreneurial venture could increase Geisinger's competitiveness by selling services back to Geisinger for less than it would otherwise cost Geisinger to provide them. 


\section{Recruiting a leader}

Geisinger's CEO believed that "scaling and generalizing" was fundamentally different from innovation. As a result, in keeping with Christensen and Raynor's recommendation, ${ }^{2}$ Geisinger's CEO decided that, in order to increase the likelihood that Geisinger's "scaling and generalizing" efforts would be successful, he should recruit someone who had experience building businesses that had scaled and generalized innovations similar to those Geisinger had produced, rather than assigning responsibility for scaling and generalizing to a GHS employee who lacked experience with scaling and generalizing and already had a full load of responsibilities.

\section{Deciding what to focus on}

Once that individual had been recruited, Geisinger's CEO recommended that that individual spend 3-6 months learning about Geisinger without being encumbered by operating responsibilities. That "discovery" process was aimed at identifying the key ingredients in Geisinger's "secret sauce", determining which of those ingredients were exportable to other delivery systems (eg, was a particular ingredient largely dependent upon an outstanding performer at Geisinger for whom it would be difficult to find or create a replica, and was there adequate documentation regarding how particular processes and technologies worked?), and determining which of those exportable capabilities could not only be scaled but also potentially be refashioned to be more efficient.

Many ingredients in Geisinger's secret sauce were identified. Examples include the following:

1. A substantial amount of customization of Geisinger's EHR, which was installed beginning in 1996. The customization included a variety of things, most notably programming that made "the right thing to do", the easiest thing for a clinician to do, various types of work flow facilitation and automation, and clinical decision support.

2. Software programs that operate outside of Geisinger's EHR but are able to exchange information with Geisinger's EHR that Geisinger clinicians and health information technology experts had developed to further enhance work flow and provide clinical decision support.

3. A data warehouse that housed data that Geisinger extracted on a nightly basis from its EHR, as well as claims data related to the approximately $45 \%$ of lives enrolled in Geisinger Health Plan who received their care from the Geisinger Clinical Enterprise.
4. An extensive set of data analytic algorithms and report templates that Geisinger employed to help manage the care of individual patients and populations of people, and help manage the operations of GHS and its various component entities.

5. Evidence-based protocols related to the performance of various types of acute care (eg, surgery and other interventional procedures, and hospitalizations for acute decompensations of chronic diseases, such as congestive heart failure and chronic obstructive pulmonary disease), as well as the outpatient management of several chronic diseases, such as diabetes, coronary artery disease, anemia secondary to chronic renal disease, and optimization of biological therapeutics used to treat hepatitis $\mathrm{C}$, multiple sclerosis, inflammatory bowel disease, and psoriasis. ${ }^{7-9}$ The distinctive features of these ProvenCare ${ }^{\circledR}$ modules were not the evidence-based guidelines on which they were based, which were in the public domain; they were the way Geisinger had integrated those protocols into clinical work flow, so they were complied with on a reliable basis and the way Geisinger monitored and reported to providers on compliance with the protocols.

6. Management designs, such as clinical service lines with a dyad (physician and administrator partner) or a triad (physician, administrator, and financial management partners).

7. Various strategies for redesigning the way care is delivered, including formation of care teams, with each member of the team operating "at the top of their license", elimination of unnecessary processes, and automation of as many processes as possible.

8. A design for an advanced patient-centered medical home (PCMH) that includes use of specially trained and equipped case managers embedded within those practices and monthly medical home meetings in which the results of data analyses are used to target care management efforts on patients and issues that will yield the greatest return. ${ }^{5,10}$

9. A curriculum and program for training case managers.

A decision was made to focus GHS' scaling and generalizing efforts initially on three things - assisting other health systems in the implementation of some of these capabilities, provision of population health data analytic services to other health systems, and provision of care managers to other health systems to either provide care management services on their behalf or train and supervise other health systems' own personnel to be effective care managers. A fourth goal was to develop software programs based on routines Geisinger 
had incorporated into its EHR or on software programs Geisinger had developed that operated outside of, but in coordination with, its EHR that would interoperate with EHRs used by other health systems, but it was not clear that this would be feasible, or if it were, within what time frame it could be done.

\section{Recognition of the difference between intellectual property and products and services that can be commercialized}

Many people and entities grossly underestimate the amount of expertise, work, and funds required to transform ideas, processes, and product prototypes into products and services that can be sold in a competitive marketplace, as well as the amount of effort required to keep those products and services up to date. GHS recognized that although it was a superb provider of health care, innovative, and an ideal laboratory for testing and refining innovations, it lacked the expertise and experience needed to develop and market products and services for other health systems. It therefore decided that, in order to realize an economic return from the innovations it had developed, it would need to recruit a group of employees who had skill sets that differed substantially from those required to operate a health care delivery system.

\section{Rationale for a stand-alone entity}

The next decision was whether to create a new entity within or outside of GHS to perform this "productization and commercialization". Geisinger had created several new entities in the past, some of which were for-profit, but all of which operated as wholly owned subsidiaries of GHS and were based in Danville, PA. Several considerations led in this case to a decision to create a separate, free-standing entity to commercialize GHS' intellectual property (IP).

The first was that Geisinger's main focus was on delivering health care to people in Pennsylvania. Although "scaling and generalizing" had been added to GHS' mission, it was clear that "scaling and generalizing" was a secondary priority compared to providing health care services to people in Pennsylvania. Second, GHS employees were already working as hard as they could in their day jobs. Consequently, "scaling and generalizing" could not just be added to their job description if the effort was going to be successful. As a result, new personnel would need to be recruited who would focus exclusively on scaling and generalizing, selling products and services, and supporting customers. Third, the new entity would need to control its own resources, rather than compete for resources with other parts of Geisinger. Finally, because target customers had to have confidence that a new entity would reliably provide the products, services, and support that they were expecting, it was felt that a free-standing entity would have greater credibility in this regard than an entity that was established within GHS.

\section{Rationale for for-profit entity}

Although the mission-related goals of launching a new entity to help other health care delivery systems improve their performance could be realized via a not-for-profit or for-profit entity, the desire to create a meaningful financial return to Geisinger from the effort and investment it has made in developing innovative approaches to delivering health care could only be fulfilled through a for-profit entity. In addition, Geisinger recognized that it would likely be easier - and require less cash - to recruit and maintain the types and caliber of talent required for a new entrepreneurial venture to be successful if the venture were a for-profit entity in which employees had an equity interest.

\section{Rationales for external funding}

Although GHS' Board was willing to invest the capital that the new entrepreneurial venture was thought to require, a decision was made to seek capital from an outside investor for several reasons. First, Geisinger wanted outside affirmation that the business plan that had been developed for the new venture was sound and worthy of investment. Second, it was thought that, in contrast to an investment by Geisinger, which is a not-for-profit entity that is mission oriented, an investment by a venture capital firm would ensure that the new venture would be run with business-like discipline. Third, venture capital firms bring substantial subject matter expertise and experience (eg, in health information technology), as well as connections to networks of individuals and companies that increase the likelihood that a start-up will be successful. Fourth, venture capital firms bring business acumen and expertise in selling companies or taking them public. Finally, by obtaining an investment from a third party, Geisinger reduced its financial risk.

\section{License agreement}

Geisinger made several contributions to the new venture, which was named $x G$ Health Solutions (xG Health). (The " $G$ " is the Geisinger " $G$ "; the " $x$ " was meant to reflect the fact that the IP was coming "out of" Geisinger, as well as the expectation that the IP would accelerate other health care delivery systems" transition from "volume" to "value" orientation.) 
Geisinger's contributions to $x G$ Health included: 1) some start-up capital, 2) the Geisinger Consulting Group, a small for-profit consulting firm that Geisinger had started several years previously, 3) a license agreement, and 4) several subcontracts which the Geisinger Health Plan entered into with $\mathrm{xG}$ Health.

By design, the license agreement had several key components. First, Geisinger licensed to $\mathrm{xG}$ Health not only all of its "health care performance improvement IP" that existed as of January 31, 2013 but also all that it developed over the succeeding 10 years. This decision was important for both pragmatic and valuation reasons. From a pragmatic perspective, Geisinger recognized that much, if not all, of the health care performance improvement IP that Geisinger had developed as of January 31, 2013 would be modified or improved in an iterative fashion over time. As a result, had Geisinger not licensed those improvements and derivative works to $\mathrm{xG}$ Health as part of the initial transaction, $x G$ Health would have had to negotiate new license agreements every time an improvement was made or a derivative work was developed. This would not only have been extraordinarily time consuming but also would have created substantial uncertainty for potential investors regarding whether $\mathrm{xG}$ Health or some other entity would get access to important clinically relevant updates to, as well as improvements in existing IP over time, as well as regarding the cost that would be required to obtain a license to such IP enhancements. Although GHS Board recognized that it could not possibly know what IP GHS would produce over the succeeding decade, and hence what the value of it might be, it also recognized that there would be substantial administrative costs related to finding potential commercialization partners and negotiating license agreements with them if it decided to license each innovation individually, rather than licensing all of them to $\mathrm{xG}$ Health. The GHS' Board and leadership therefore decided to "go all in" on $x G$ Health, with the expectation that $x G$ Health would be as good an entity as any other to transform Geisinger innovations into commercializable products, and market, sell, and support them successfully.

Second, the license was perpetual. That is, there was no limit on the time during which $\mathrm{xG}$ Health had a right to commercialize the IP Geisinger licensed to it. This was particularly important to an outside investor, who inevitably would want to realize an economic return on its investment in $x G$ Health. To do so, the terms of the license between GHS and $x G$ Health had to allow $x G$ Health to retain its license rights over the long term - even if it were acquired by another entity.
Third, the license had a non-compete provision. This provision also was important to securing an outside investment at a favorable valuation because the likelihood of $x G$ Health being successful financially was low if Geisinger could use its IP to compete against $\mathrm{xG}$ Health. This concern was more than theoretical, since the Geisinger Health Plan had begun to market its insurance, third-party administrator, and population health management services to self-insured health care delivery systems outside of Pennsylvania. By GHS agreeing to not compete with $\mathrm{xG}$ Health in the population health management space outside of the parts of Pennsylvania in which GHS operated, Geisinger would be able to present a single face to the marketplace for such services, thus eliminating potential confusion regarding the differences between the products and services offered by different Geisinger-related entities, avoiding the inefficiency that would occur if efforts to sell similar services or products were mounted by different parts of Geisinger, and promoting collaboration and coordination among personnel at Geisinger and $\mathrm{xG}$ Health.

Fourth, the license was exclusive for a minimum of 4 years, and would be exclusive for 10 years if $x G$ Health achieved pre-specified financial milestones.

Finally, the license was reciprocal, ie, Geisinger received a royalty-free license to derivative works and new IP that $x G$ Health developed.

\section{Securing external capital}

Geisinger engaged JP Morgan, which had been its banker for more than 30 years, to vet the $\mathrm{xG}$ Health business plan and assist it in raising capital. The result of that process was a \$40 million investment from Oak Investment Partners. $x G$ Health was formally launched on February 1, 2013 as an independent, for-profit company.

\section{Location of the company}

Columbia, MD was selected as xG Health's headquarters for close proximity to an airport and the Baltimore/Washington, DC area from where relevant talent could be recruited, and for reasonable proximity to Geisinger. In order to get $\mathrm{xG}$ Health off the ground quickly with a high-caliber work force, a decision was made to recruit the best possible talent regardless of where they resided, rather than try to get all new hires to move to Columbia, MD. While this decision has enabled $\mathrm{xG}$ Health to grow quickly, it makes direct interaction among employees more difficult. To overcome this challenge, $x \mathrm{G}$ Health regularly employs videoconferencing and provides frequent updates to all its personnel through quarterly conference calls and corporate newsletters. 


\section{Structure of the ongoing relationship between $\times \mathbf{G}$ Health and GHS}

Many efforts have been made to ensure that there is a close ongoing working relationship between $\mathrm{xG}$ Health and Geisinger. Several of these are worthy of mention. First, three of seven seats on $x \mathrm{G}$ Health's Board were reserved for Geisinger appointees, with Geisinger's CEO at the time of $\mathrm{xG}$ Health's founding (Dr Steele) serving as Chairman of the Board. Second, a committee with representatives from both $x G$ Health and Geisinger was established to identify pertinent IP that had been developed by either entity and manage the transfer of it to the other party. Third, another committee with representatives from both organizations was established to help coordinate and manage activities of mutual interest, as well as potential conflicts. Fourth, there has been substantial collaboration between $\mathrm{xG}$ Health and Geisinger employees to transform various types of GHS IP into products that can be exported for use at other health care delivery systems and/or that are scalable. Finally, there are contractual relationships under which each entity provides services to the other. For example, $x G$ Health pays GHS for time Geisinger clinical experts spend assisting on $\mathrm{xG}$ Health customer engagements and GHS pays $\mathrm{xG}$ Health for data analytic services that support Geisinger's Accountable Care Organization.

\section{The first 2 years}

During its first 2 years, $x G$ Health successfully transformed several types of Geisinger IP into products and services that can be exported to other health care delivery systems at scale. For example, $\mathrm{xG}$ Health has developed its own data analytic infrastructure that it is using to deliver to clinicians at several health care delivery systems the same population health management reports that are produced by Geisinger. Second, $x G$ Health has developed a multimedia online learning system that is helping it implement substantial numbers of advanced PCMHs simultaneously, train large numbers of case managers, and train multidisciplinary teams in implementation of evidence-based care protocols. Third, $x G$ Health has developed care management software that enables case managers to follow patient management protocols that are used at Geisinger. Finally, and likely of greatest significance, employees at Geisinger and $\mathrm{xG}$ Health identified a way to enable software programs developed at Geisinger over the past 18 years that heretofore have worked only with Geisinger's EHR to work in concert with EHRs at other health care delivery systems. This breakthrough will markedly enhance xG Health's ability to implement the same clinical work flows and provide the same data analytically driven clinical decision support at other provider organizations that have contributed to Geisinger's outstanding clinical and economic performance.

As a result of these and other product and service development activities, $\mathrm{xG}$ Health was providing services to more than 30 health care delivery systems across the country and in Singapore by the end of its second year, and had grown substantially in terms of both revenue and number of employees.

\section{The future}

Although, during its brief existence, $\mathrm{xG}$ Health has been fulfilling the goals that prompted Geisinger to establish it, it is too soon to know how successful $\mathrm{xG}$ Health will be or how its relationship with Geisinger will evolve. For example, if $x G$ Health needs additional capital to sustain or expand its growth, GHS will need to decide whether it wants to increase its investment in $\mathrm{xG}$ Health in order to maintain its percentage ownership in xG Health. In addition, in May of 2015, a new CEO took the helm at GHS, and it is too soon to know how, if at all, that individual will want to evolve the relationship between GHS and $x G$ Health. What is clear though is that Geisinger's judgment that a separate, but related, entrepreneurial entity was a good way to "scale and generalize" Geisinger's health care performance improvement IP has been sound so far.

\section{Disclosure}

Dr Steinberg is CEO of, and has an equity interest in, $x G$ Health Solutions. Dr Steele is Chairman of $x G$ Health Solution's Board and is expected, in addition, to become an independent contractor to, with an equity interest in, $\mathrm{xG}$ Health Solutions in July 2015. The authors report no other conflicts of interest in this work.

\section{References}

1. Christensen CM. The Innovator's Dilemma. New York: Harper Business; 2000 .

2. Christensen CM, Raynor ME. The Innovator's Solution. Creating and Sustaining Successful Growth. Boston: Harvard Business School Press; 2003

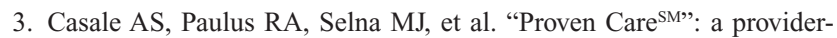
driven pay-for-performance program for acute episodic cardiac surgical care. Ann Surg. 2007;246(4):613-621.

4. Paulus R, Davis K, Steele G Jr. Continuous innovation in health care: implications of the Geisinger experience. Health Aff. 2008;27(5): 1235-1245.

5. Gilfillan RJ, Tomcavage J, Rosenthal MB, et al. Value and the medical home: effects of transformed primary care. Am J Manag Care. 2010; 16(8):607-614.

6. Steele GD, Haynes JA, Davis DE, et al. How Geisinger's advanced medical home model argues the case for rapid-cycle innovation. Health Aff. 2010;29(11):2047-2053. 
7. Maeng DD, Graham J, Graf TR, et al. Reducing long-term cost by transforming primary care: evidence from Geisinger's medical home model. Am J Manag Care. 2012;18(3):149-155.

8. Bloom F Jr, Graf T, Steele G Jr. Improved Patient Outcomes in 3 years with a System of Care for Diabetes; 2012: IOM Learning Health System Commentary Series. Institute of Medicine of the National Academies Advising the Nation-Improving Health.
9. Bloom FJ Jr, Yan X, Stewart WF, et al. Primary care diabetes bundle management: 3-year outcomes for microvascular and macrovascular events. Am J Manag Care. 2014;20(6):175-182.

10. Maeng DD, Khan N, Tomcavage J, Graf TR, Davis DE, Steele GD. Reduced acute inpatient care was largest savings component of Geisinger health system's patient-centered medical home. Health Aff (Millwood). 2015;34(4):636-644.

\section{Publish your work in this journal}

Innovation and Entrepreneurship in Health is an international, peer reviewed, open access journal publishing original research, reports, reviews and commentaries on innovation and entrepreneurship in health. Special focus will be given to the theory, process, and practice of innovation and entrepreneurship by individuals and organizations

\section{Dovepress}

within the health care context globally. The manuscript management system is completely online and includes a very quick and fair peer review system, which is all easy to use. Visit http://www.dovepress.com/ testimonials.php to read real quotes from published authors.

Submit your manuscript here: http://www.dovepress.com/innovation-and-entrepreneurship-in-health-journal 